\section{GP220 PRIORITY FOR COMPLEMENTARY FEEDING START COUNSELING AND RELATED NUTRITIONAL RECOMMENDATIONS FROM PEDIATRICIANS DURING THE FIRST YEAR OF LIFE}

${ }^{1}$ Paolo Brambilla*, 'Marina Picca, ${ }^{2}$ Gaetano Bottaro, ${ }^{1}$ Marco Giussani, ${ }^{3}$ Roberto Buzzetti, ${ }^{4}$ Paolo Becherucci. ${ }^{1}$ Società Italiana delle Cure Primarie Pediatriche, Milano, Italy; ${ }^{2}$ Società Italiana delle Cure Primarie Pediatriche, Catania, Italy; ${ }^{3}$ Epiemiologia Clinica, Bergamo, Italy; ${ }^{4}$ Società Italiana delle Cure Primarie Pediatriche, Firenze, Italy

\subsection{6/archdischild-2019-epa.279}

Introduction and aim An early education to avoid an excessive protein and salt consumption is now considered crucial for obesity and hypertension prevention. Complementary feeding (CF) practices are debated among Pediatricians, first of all about which criterion should be considered between nutritional needs (NN) or developmental readiness (DR) for CF start. The aim of the present study was to analyze if timing of $\mathrm{CF}$, modalities, and nutritional advices during the first year of life were related with the criterion considered for CF start among Pediatricians.

Methods An online Survey was conducted in march 2018 among Family Pediatricians in Italy investigating the criterion chosen for CF start, timing, method of feeding and specific dietary practices. A Good Nutritional Practice (GNP) was acknowledged to those Pediatricians who declared to give advices for meat quantity and salt consumption during the first year of life respect to those who declared to demand any decision to parents.

Results Participation rate was 43.3\% (350 of 808) among active members of the Italian Society of Primary Care Pediatricians (SICuPP), 213 of them (60.9\%) choose DR and 137 (39.1\%) $\mathrm{NN}$ as CF starting criterion. About $75 \%$ declared to counsel CF start between 5 and 6 months of age, $17 \%$ before 5 months and $8 \%$ after 6 months. Concerning CF modalities, $38 \%$ suggested Traditional pureed foods spoon feeding following written recommendations (T), 13\% suggested a sort of Baby-led weaning (B) while the majority (49\%) declared to use both according with family characteristics. Concerning specific advices, $89 \%$ declared to suggest meat quantity during the first year of life and $91 \%$ suggest to introduce added salt only after 12 months of age. A GPN was followed by $85 \%$ of them.

NN Pediatricians had a significantly earlier CF starting, an higher use of $\mathrm{T}$ modality, meat quantity and added salt advices, and followed more frequently a GNP respect to DR Pediatricians (all p<0,0001).

Conclusions Our data suggest that the criterion most considered for CF start might be associated with timing, modality and nutritional advices during the first year of life. Pediatricians following the developmental readiness position for $\mathrm{CF}$ start could less frequently give to parents adequate nutritional advices for hypertension and obesity prevention. An effort aimed to integrate new $\mathrm{CF}$ practices with adequate nutritional recommendations should be strongly encouraged.

\section{GP221 INFLUENCE OF CONTROLLED PHYSICAL ACTIVITY ON SERUM ADIPOKINES CONCENTRATION IN OBESE CHILDREN}

\footnotetext{
${ }^{1}$ Anna Wasilewska* ${ }^{1}$ Piotr Protas, ${ }^{1}$ Agnieszka Rybi-Szuminska, ${ }^{1}$ Katarzyna Taranta-Janusz, ${ }^{2}$ Marian Stelmach, ${ }^{1}$ Elżbieta Kuroczycka-Saniutycz, ${ }^{1}$ Marta Lemiesz. 'Medical University of Bialystok, Bialystok, Poland; '2Pope John Paul II State School of Higher Education, Biała Podlaska, Poland
}

10.1136/archdischild-2019-epa.280
Introduction The number of obese people is increasing, and its negative impact on the people's health is significant. The relationship between physical activity and obesity is still under investigation. One of components responsible for the metabolism are adipokines such as adropin or adiponectin. The purpose of this study was to investigate, whether the controlled physical activity affects the concentrations of adipokines and may play role in treatment of obesity in children.

Material and Methods 34 obese children aged 5-18 years were involved to the dynamic prospective study. The reference group consisted of 16 healthy children. The participants were informed about recommended physical activity, adjusted for sex, age, and degree of overweight. They were equipped with exercise recorder for a period of 8 weeks. Before start of the study and after 8 weeks of effort, has been made anthropometric measurements, electrical bioimpedance and blood serum was collected. Adropin and adiponectin concentrations in serum were determined by ELISA.

Results In the study group, 22 children decreased BMI Zscore. Average BMI Z-score has decreased from $2.75 \pm 0.43$ at baseline to $2.51 \pm 0.31$ at the end of the study $(p<0.05)$. In the whole study group, there was no statistical significant differences in the concentrations of adiponectin and adropin compared between study points and the control group. In contrast, significantly increase the concentration of adropin after 8 weeks, in group of patients who have lowered their BMI Z-Score $(38.84 \pm 20.29$ vs. $64.54 \pm 40.45 \mathrm{pg} / \mathrm{ml}, \mathrm{p}<0.01)$.

Conclusions Controlled physical activity leads to reduction of obesity in children and increases serum adropine concentration, which may play role in prevention of obesity complications.

\section{GP222 CLINICAL AND METABOLIC PARAMETERS IN GIRLS- CARRIERS OF LEPR RS1137100 WITH ANDROID AND GYNOID OBESITY}

Ekaterina Sheneman, Tatyana Bairova, Ksenia levleva, Lyubov Rychkova*, Olga Bugun. Scientific Centre for Family Health and Human Reproduction Problems, Irkutsk, Russian Federation

\subsection{6/archdischild-2019-epa.281}

Leptin is a peptide hormone of adipose tissue regulating energy metabolism. Numerous studies indicate LEPR gene contribution to obesity. There are no data on the role of LEPR gene rs1137100 polymorphism in development of android and gynoid types of obesity.

Aim To compare the clinical and metabolic parameters in adolescent girls with android and gynoid obesity types - carriers of different genotypes of LEPR gene rs1137100 polymorphism.

Materials and Methods We examined 88 Caucasian girls (aged $15.8 \pm 0.09$ ) with body mass index SDS (SDS BMI) $\geq 2.0$, living in Eastern Siberia (Russia). The SDS BMI $\geq 2.0$ girls were divided in 2 groups: 41 girls (aged 15.4 \pm 0.1 ) with android obesity (waist measurement $97.3 \pm 1.7 \%$ ) and 47 girls with gynoid (waist measurement $86.1 \pm 15.1 \%$ ), (aged 15.5 \pm 0.1 ). We measured circumference and skinfold thickness in following areas: blades, chest, belly, thighs, triceps, and biceps. We investigated metabolism parameters: glucose, insulin, leptin in serum; insulin sensitivity index (HOMA-IR) was calculated. We tested frequency of LEPR rs1137100 polymorphism in girls with android and gynoid obesity. 
Results Primarily we compared thickness of subcutaneous adipose tissue in girls-carriers of different genotypes in both groups. Girls with android obesity - carriers of AA genotype, thickness of subcutaneous adipose tissue in breast was 1.1 $\pm 0.2 \mathrm{~cm}$; AG genotype - $0.94 \pm 0.2 \mathrm{~cm}$; GG-genotype - 0.93 $\pm 0.2 \mathrm{~cm}$ ( $\left.\mathrm{p}^{\mathrm{AA}-\mathrm{AG} ; \mathrm{AA}-\mathrm{GG}}=0.04\right)$. Metabolism parameters: insulin in carriers of AA genotype was 25.2 \pm 13.6 ; AG genotype 15.5 \pm 8.3 ; GG genotype $-18.1 \pm 11.4\left(\mathrm{p}^{\mathrm{AA}-\mathrm{AG}}=0.01\right)$; HOMAIR, $6.1 \pm 3.6 ; 3.4 \pm 2.0 ; 4.4 \pm 3.3 \quad\left(\mathrm{p}^{\mathrm{AA}-\mathrm{AG}}=0.01\right)$, respectively. Leptin without statistically significant differences was elevated in AA genotype carriers $67.1 \pm 25.1$, in contrast to carriers of AG and GG genotypes: $54 \pm 26.6 ; 50.8 \pm 26.5$, respectively. In girls with a gynoid obesity - carriers of AA genotype, thickness of subcutaneous adipose tissue in thighs was $2.0 \pm 0.2 \mathrm{~cm}$; $A G$ genotype - $2.1 \pm 0.3 \mathrm{~cm}$; GG-genotype $-2.5 \pm 0.8 \mathrm{~cm} \quad\left(\mathrm{p}^{\mathrm{AA}-}\right.$ $\mathrm{GG}=0.03)$. Metabolism parameters: insulin in AA genotype carriers was 13.4 \pm 5.1 ; AG genotype - 11.8 \pm 6.3 ; GG genotype $21 \pm 14.2\left(\mathrm{p}^{\mathrm{AA}-\mathrm{GG}}=0.04 ; \mathrm{p}^{\mathrm{AG}-\mathrm{GG}}=0.02\right)$. Leptin $34.5 \pm 15.7 ; 32.2$ $\pm 16.3 ; 55.8 \pm 19.7\left(\mathrm{p}^{\mathrm{AA}-\mathrm{GG}}=0.005 ; \mathrm{p}^{\mathrm{AG}-\mathrm{GG}}=0.003\right)$ respectively. HOMA-IR in carriers of AA genotype was $3.0 \pm 1.6$; AG genotype - 2.6 \pm 1.4 ; GG-genotype - $4.5 \pm 3.2\left(\mathrm{p}^{\mathrm{AG}-\mathrm{GG}}=0.04\right)$.

Conclusions In girls of android morphotype, the carriage of A-allele is associated with carbohydrate and energy metabolism disorders, and is a risk marker of excess fat deposition in chest area. For a gynoid morphotype, G-allele is a risk marker and is associated with excessive fat deposition in the thighs, as well as with carbohydrate and energy metabolism disorders.

\section{GP223 CREATINE DEFICIENCY DISORDERS THE ALL-IRELAND EXPERIENCE}

${ }^{1}$ Caoimhe Howard*, 'AA Monavari, 'Ina Knerr, 'Gregory Pastores, ${ }^{2}$ Siobhan O'Sullivan, ${ }^{1}$ Ellen Crushell. ${ }^{1}$ National Centre for Inherited Metabolic Disorders, Temple Street Children's University Hospital, Dublin, Ireland; '2Department of Paediatrics, Royal Belfast Hospital for sick Children, Belfast, UK

\subsection{6/archdischild-2019-epa.282}

Background Cerebral creatine deficiency is caused by rare inherited disorders of either creatine biosynthesis (i.e. guanidinoacetate methyltransferase (GAMT) and arginine:glycine amidinotransferase (AGAT) deficiency), or creatine transport (i.e. creatine transporter deficiency, CRTR). Clinical features include variable hypotonia, speech delay (often the dominant feature), seizures, extrapyramidal signs and behavioural issues. Patients may have feeding issues and low weight. There is some evidence that early treatment of these disorders is successful, particularly in GAMT and AGAT deficiency.

Method A retrospective chart review was undertaken of all patients who have attended the national metabolic referral centres at Dublin and Belfast with creatine deficiency. Details recorded included history, clinical findings, treatments and results of laboratory, genetic and radiological investigations.

Results Six patients with current ages between 5 and 29 years were identified with a confirmed disorder of creatine metabolism - four male patients with X-linked CRTR and two female siblings with autosomal recessive GAMT deficiency. Initial diagnosis was made on urine biochemistry (analysis of creatine/creatinine and guanidinoacetate) in two patients, on MR spectroscopy in three patients (creatine peak significantly reduced) and via exome sequencing in one patient. All cases were confirmed biochemically and genetically. Despite onset of symptoms before the age of 2 years, mean age at diagnosis was 7.5 years, with range from 20 months to 17 years.
Six patients had significant developmental delay, particularly in the domains of speech and behaviour. Three of the patients have weights $<9$ th centile. Five of the patients had documented head circumferences, and all were normocephalic proportional to height and weight. Both patients with GAMT deficiency had epilepsy which responded to treatment with creatine and ornithine. Three patients with CRTR also had epilepsy. Two patients with CRTR have been treated with creatine and creatine/arginine/glycine/S-adenosylmethionine in combination, without notable effect on clinical symptoms or MR spectroscopy findings, which is in keeping with expectations for this condition.

The four patients with CRTR are hemizygous for pathogenic mutations in the SLC6A8 gene, de novo in two patients and maternally inherited in another, one has not had parental testing. The siblings with GAMT deficiency are compound heterozygous for mutations in the GAMT gene.

Conclusion Although rare in Ireland, these treatable disorders are likely under-diagnosed. In a patient with developmental delay (particularly speech impairment) and behavioural difficulties, consideration should be given to sending a urine sample for analysis of creatine/creatinine ratio and guanidinoacetate, particularly if there is comorbid epilepsy.

\section{GP224 MULTISYSTEM MITOCHONDRIAL DISEASES IN CHILDREN WITH MATERNALLY INHERITED COMPLEX I DEFICIENCY}

Tereza Danhelovska, Hana Kolarova, Jan Langer, Kamila Berankova, Hana Hansikova, Marketa Tesarova, Tomas Honzik, Jiri Zeman*. Department of Paediatrics, First Faculty of Medicine, Charles University and General University Hospital, Prague, Czech Republic

\subsection{6/archdischild-2019-epa.283}

Mitochondrial disorders (MD) in childhood represent a heterogeneous group of disease. The most common cause of MD is respiratory chain complex I (CI) deficiency, which may be caused by mutations in either nuclear or the mitochondrial DNA (mtDNA). In the cohort of 106 unrelated families with mtDNA mutations from our region with 10,5 million of inhabitants, the multisystem MD due mtDNA mutations in MT-ND genes for structural subunits of CI were recognized in 12 families with 13 affected children.

Results In the group of 13 patients, altogether 8 different heteroplasmic mtDNA mutations in MT-ND genes were found. Mutations in MT-ND5 gene were most frequent including one novel mutation m.13091T $>$ C. Six children with the mutation heteroplasmy $>60 \%$ had Leigh syndrome and significantly worse prognosis than five patients with heteroplasmy $<60 \%$, who developed MELAS syndrome with stroke-like episodes. In last two children, the diseases started with optic neuropathy but both children transitioned later to multisystem diseases compatible with MELAS syndrome. The activities of CI in isolated muscle mitochondria were decreased in most patients and analyses with $\left[1-{ }^{14} \mathrm{C}\right]$ pyruvate, $\left[\mathrm{U}-{ }^{14} \mathrm{C}\right]$ malate and $\left[1,4-{ }^{14} \mathrm{C}\right]$ succinate substrates revealed decreased $\mathrm{CO}_{2}$ production in some patients.

Conclusions Children with the multisystem MD due to $\mathrm{CI}$ deficiency and heteroplasmic mtDNA mutations usually develop Leigh or MELAS syndromes and represent approximately $11 \%$ of families with maternally inherited MD diagnosed in our region. Early onset of the disease and higher level of heteroplasmy of mtDNA mutations resulted in Leigh 